\title{
Differential expression of claudin- 4 between intestinal and diffuse-type gastric cancer
}

\author{
WEN-LING KUO ${ }^{1}$, LI-YU LEE ${ }^{2}$, CHI-MING WU ${ }^{3}$, CHEE-CHAN WANG ${ }^{4}$, JAU-SONG YU $^{5}$, \\ YING LIANG $^{1}$, CHIH-HONG LO ${ }^{1}$, KUO-HAO HUANG ${ }^{5}$ and TSANN-LONG HWANG ${ }^{1}$
}

\begin{abstract}
Departments of ${ }^{1}$ Surgery, ${ }^{2}$ Pathology, Chang Gung Memorial Hospital; ${ }^{3}$ Biotechnology, ${ }^{4}$ Cosmetic Science, Vanung University; ${ }^{5}$ Graduate Institute of Basic Medical Science, Chang Gung University, Tao-Yuan, Taiwan
\end{abstract}

Received May 11, 2006; Accepted June 23, 2006

\begin{abstract}
Our previous microarray analysis of gastric cancer found that claudin- 4 was differentially expressed between intestinal-type gastric cancer (IGC) and diffuse-type gastric cancer (DGC). Claudin-4 is a member of a large family of transmembrane proteins, claudins, essential in the formation and maintenance of tight junctions. To explore the roles of claudin-4 in the two histologically distinct types of gastric cancer, we selected 45 IGC and 48 DGC cases and then analyzed the expression of the protein using immunohistochemistry. We found that the overexpression of claudin-4 was greater in IGC than in DGC. A trend was observed between the overexpression of claudin-4 and lymph node metastasis, however, this association was not statistically significant. The results showed that the expression of claudin-4 was lower in DGC. Possibly it played a role in determining the diffuse phenotype and loose cohesion of cells in DGC in a similar manner as E-cadherin.
\end{abstract}

\section{Introduction}

Gastric cancer is the second most frequent cause of cancer death in the world after lung cancer (1). According to Lauren's classification, gastric cancer can be divided into two histologically distinct types, each of which accounts for half of the cases: Intestinal-type gastric cancer (IGC) and diffuse-type gastric cancer (DGC) (2). IGC, the predominant type of tumor in high-risk areas, has a glandular pattern and is usually accompanied by papillary formation or solid components (2). DGC, in contrast, consists of poorly cohesive cells diffusely infiltrating the gastric wall with little or no gland formation (2). A special subgroup of this type is the so-called signet ring cell carcinoma, in which the cell nucleus is pushed against

Correspondence to: Dr Tsann-Long Hwang, Department of Surgery, Chang Gung Memorial Hospital, No. 5 Fu-Hsing Street, 333 Tao-Yuan, Taiwan

E-mail: hwangtl@adm.cgmh.org.tw

Key words: intestinal-type gastric cancer, diffuse-type gastric cancer, claudin-4, immunohistochemistry, E-cadherin the cell membrane creating a classical signet appearance due to an expanded, globoid, optically clear cytoplasm. IGC and DGC may result from the transformation of different epithelial cells or distinct molecular changes in common cell types (3).

Over the past decade, many studies have clearly demonstrated that the combination of molecular changes differs between IGC and DGC, suggesting that they have unique genetic alterations (4-6). Alterations in specific genes that play important roles in diverse cellular functions such as cell adhesion, signal transduction, differentiation, development or DNA repair have been identified $(7,8)$. With regard to genetic alterations in tumor suppressor genes or oncogenes, gastric cancer is no exception. Inactivation due to loss of heterozygosity $(\mathrm{LOH})$ and/or mutation of p53, APC and DCC have been reported in gastric cancer. Mutation of p53 was detected in $\sim 30 \%$ of gastric cancers independent of the histological subtype $(9,10)$. Up to $60 \%$ of the IGC cases but only $30 \%$ of DGC have a mutation/LOH of the APC gene (11). LOH of DCC has been detected in $50 \%$ of IGC cases, whereas in DGC, LOH is absent (12). The met protooncogene codes for the hepatocyte growth factor receptor which is preferentially amplified and overexpressed in DGC (13). Other growth factor and receptor signal systems that may be altered include EGFR, TGF- $\alpha$ and K-sam (14). Adhesion molecules such as $\beta$-catenin have been detected in $30 \%$ of IGC mutations but are absent in DGC (15). Ecadherin is the binding partner of $\beta$-catenin and plays a crucial role in establishing the structural integrity of epithelial tissues. E-cadherin mutations have been detected in $50 \%$ of DGC but absent in IGC (16).

Claudins are a large family of transmembrane proteins essential in the formation and maintenance of tight junctions (17). Tight junctions in epithelial cells provide a selective barrier and establish cellular polarity (18-20). These structures are typically lost in cancer and this loss may contribute to the invasive and metastatic phenotype of tumor cells (21-24). Using cDNA microarray analysis, we have previously shown that claudin-4 is differentially expressed between IGC and DGC (25). Claudin-4 was more overexpressed in IGC than in DGC. In the present study, we used 45 IGC and 48 DGC specimens to examine whether the immunohistochemical staining of claudin-4 is different between the two types of gastric cancer. 
Table I. Clinicopathological parameters of patient samples included for claudin-4 immunohistochemical analysis.

\begin{tabular}{|c|c|c|c|}
\hline Parameters & IGC & DGC & P-value \\
\hline Total & 45 & 48 & \\
\hline \multicolumn{4}{|l|}{ Gender } \\
\hline Male & 30 & 26 & $>0.05$ \\
\hline Female & 15 & 22 & \\
\hline \multicolumn{4}{|l|}{ Age } \\
\hline Mean \pm SD & $64 \pm 9.4$ & $57 \pm 14.2$ & 0.00704 \\
\hline$\leq 60$ & 17 & 28 & 0.047 \\
\hline$>60$ & 28 & 20 & \\
\hline \multicolumn{4}{|c|}{ Depth of wall invasion } \\
\hline $\mathrm{T} 1$ & 15 & 8 & $>0.05$ \\
\hline $\mathrm{T} 2$ & 6 & 5 & \\
\hline $\mathrm{T} 3$ & 19 & 28 & \\
\hline $\mathrm{T} 4$ & 5 & 7 & \\
\hline \multicolumn{4}{|c|}{ Lymph node metastasis } \\
\hline N0 & 19 & 14 & $>0.05$ \\
\hline N1 & 15 & 15 & \\
\hline $\mathrm{N} 2$ & 7 & 15 & \\
\hline N3 & 4 & 4 & \\
\hline \multicolumn{4}{|l|}{ Differentiation } \\
\hline Well & 8 & 2 & $>0.05$ \\
\hline Moderate & 14 & 18 & \\
\hline Poor & 23 & 28 & \\
\hline
\end{tabular}

\section{Patients and methods}

Patients and specimens. Tissues specimens $(\mathrm{N}=93)$ were collected from patients with gastric cancer requiring subtotal or total gastrectomy resection in Chang Gung Memorial Hospital (CGMH) in Taiwan. All operations were performed between January 2000 and December 2001. Written informed consent was obtained before collection and this study was approved by the Institutional Review Board. Table I summarizes the clinical parameters of patients with gastric cancer. All specimens were divided into two groups according to Lauren's classification, IGC ( $\mathrm{N}=45,30$ men and 15 women) and DGC ( $\mathrm{N}=48,26$ men and 22 women). The mean ages of IGC and DGC patients were 64 and 57 years, respectively. Pathological TNM stages were obtained from clinical records. All tissue specimens were formalin-fixed and paraffinembedded. Formalin-fixed tissue sections were stained with $\mathrm{H} \& \mathrm{E}$ and classified by a pathologist. These results were compared with the pathology record from CGMH. Final pathology was determined by consensus and reviewed if necessary.

Immunohistochemistry. The tissue block were constructed according to the method of Schraml et al (26) and the best representative morphological areas of the tumors were used in this study. The specimen sections were deparaffinized, treated with $3 \%$ hydrogen peroxide and microwaved after pretreatment in $10 \mathrm{mM}$ citric acid to retrieve antigenicity. The sections were incubated with blocking solution containing PBS and $1 \%$ bovine serum album for $20 \mathrm{~min}$ at room temperature, and then incubated with anti-claudin-4 antibody (goat anti-human polyclonal antibody from Santa Cruz Biotechnology) overnight at $4^{\circ} \mathrm{C}$. After washing $4 \mathrm{x}$ with TBS, the sections were incubated with secondary antibody (rabbit anti-goat IgG, Santa Cruz Biotechnology). The immuno-complex was visualized by the immonoglobulin enzyme bridge technique using the Dako LSAB 2 System, HRP kit (Dako Co., Carpinteria, CA, USA) with 3, 3' diaminobenzidine tetrachloride as a substrate. The sections were lightly counterstained with hematoxylin, dehydrated with graded alcohols, cleared with xylene and mounted with a coverslip.

Scoring of the immunostaining. The immunostaining results were scored as follows according to a previous report (27). The immunostaining reaction was evaluated by subjective assessments of the median staining intensity ( 0 , no stain; 1 , weak; 2 , moderate; 3 , strong) and by the fraction of stained cells in percentage categories $(0,0-9 \% ; 1,10-49 \% ; 2,50$ $89 \%$; and $3, \geq 90 \%$ ). This scoring system was previously shown to be reproducible (28). Claudin-4 immunoreactivity was classified as negative if $<10 \%$ of the cells were stained. The scores of 0 to 3 were obtained as follows: Percentage categories and staining were each ranked as indicated above. The ranks for percentage and staining intensity were multiplied by each other, divided by 3 , and rounded up to the nearest whole number (28). The results of immunostaining in the tumor and normal tissues were divided into three groups: strong (rank of tumor tissue >rank of normal tissue), equal (rank of tumor tissue $=$ rank of normal tissue), and weak (rank of tumor tissue <rank of normal tissue) (Fig. 2).

Statistical analysis. Chi-square or Fisher's exact test were used to test for an association between claudin-4 expression and the clinicopathological parameters. The level of significance was set at 0.05 . All reported p-values were two-sided. The data analyses were carried out using SAS statistics package (version 8.1 for windows; SAS Institute, Inc., Cary, NC).

\section{Results}

Expression of claudin-4 in IGC and DGC. The H\&E staining of IGC and DGC is shown in Fig. 1A and B, respectively. IGC has a glandular pattern and is usually accompanied by papillary formation or solid components. DGC, in contrast, consists of poorly cohesive cells diffusely infiltrating the gastric wall with little or no gland formation. A special subgroup of this type is the so-called signet ring cell carcinoma, in which the cell nucleus is pushed against the cell membrane creating a classical signet appearance due to an expanded, globoid, optically clear cytoplasm. The results of immunostaining in tumor and normal tissues were divided into three groups: strong (rank of tumor tissue >rank of normal tissue) (Fig. 2A and B), equal (rank of tumor tissue = rank of normal 

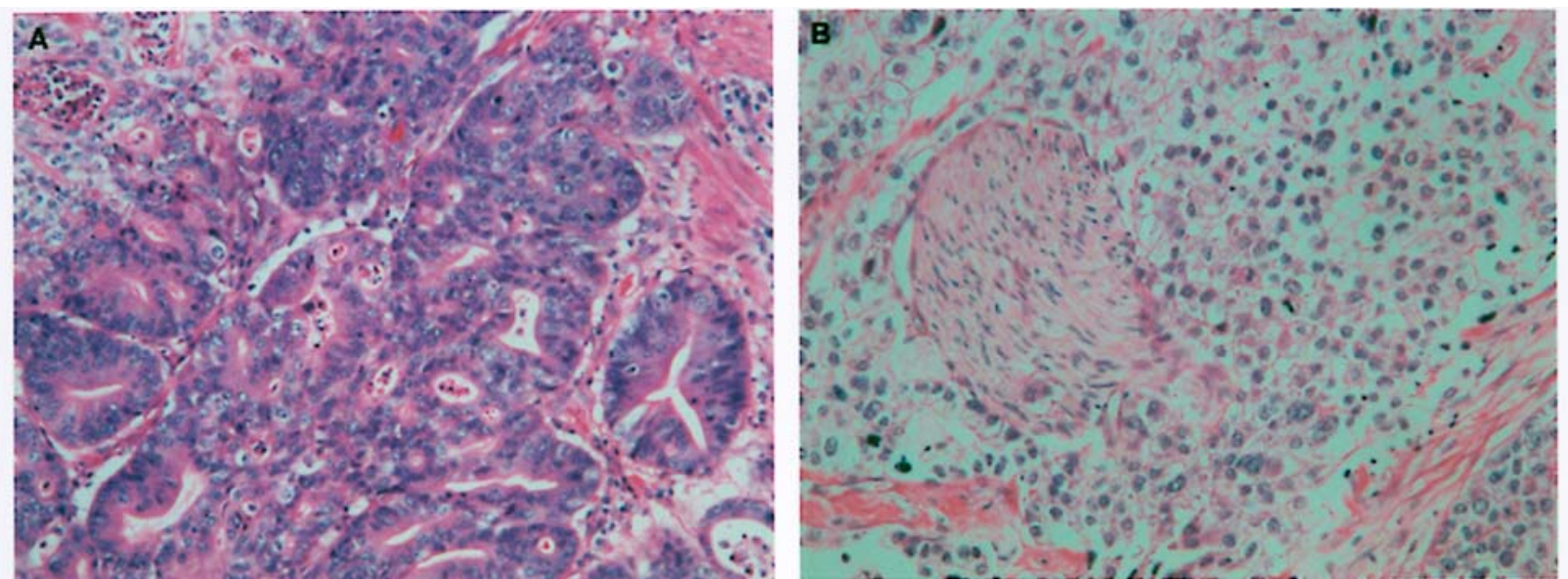

Figure 1. The H\&E staining of IGC and DGC (magnification x200). (A), IGC and (B), DGC.

IGC
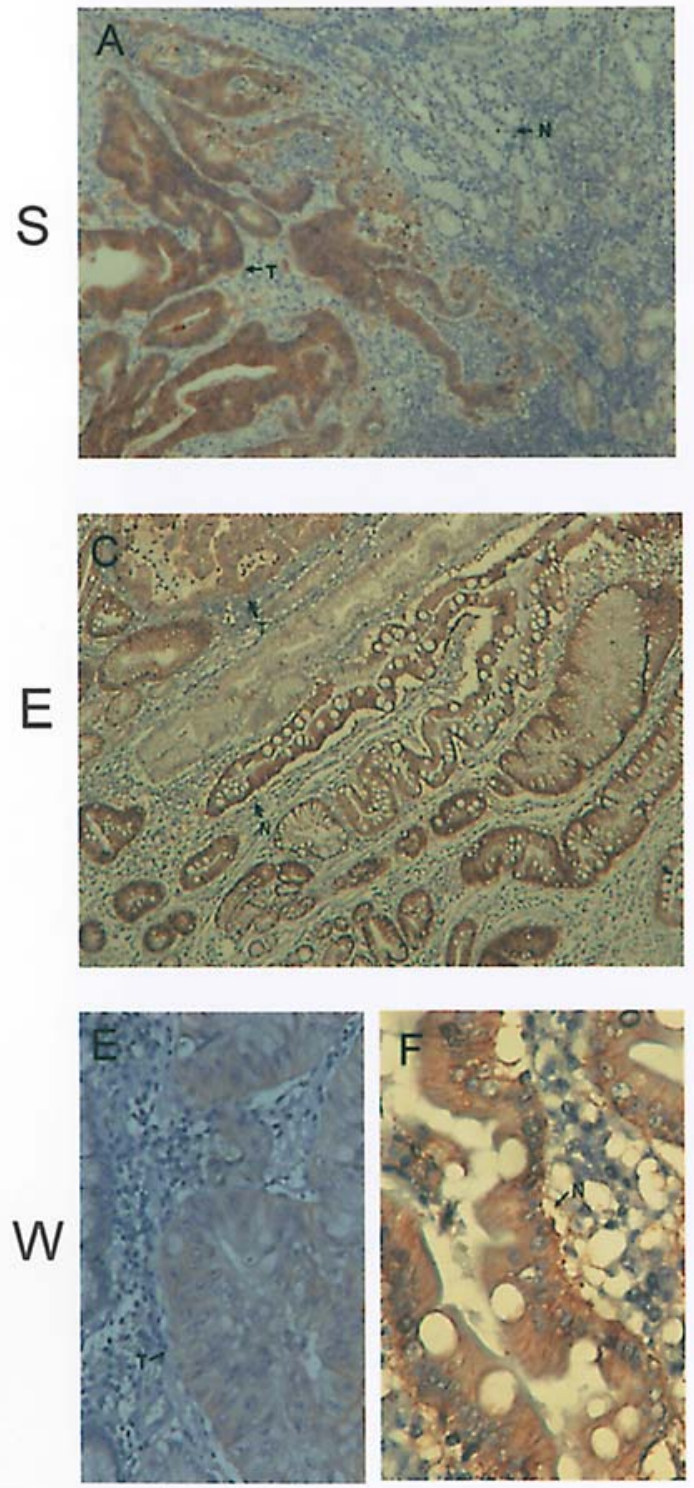

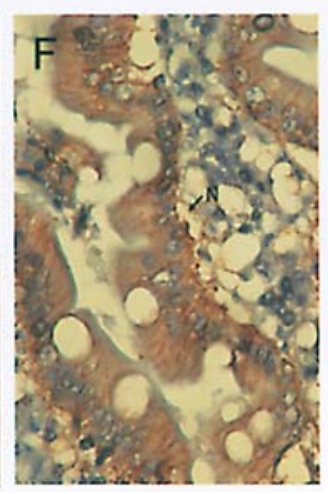

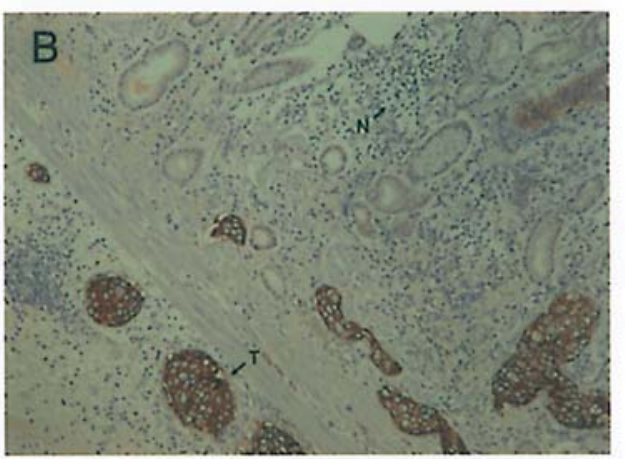
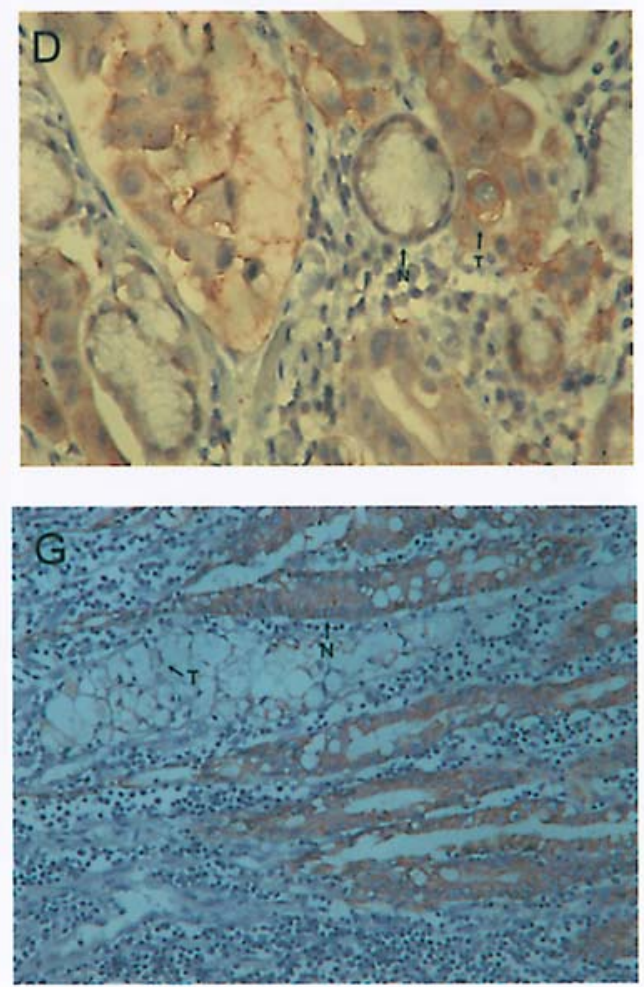

Figure 2. Immunohistochemistry of claudin-4 in IGC and DGC. The overexpression of claudin-4 in IGC and DGC greater than in normal tissues is shown in (A) (magnification $\mathrm{x} 100$ ) and (B) (magnification x100), respectively. The expression of claudin-4 in IGC and DGC equal to that in normal tissues is shown in (C) (magnification $\mathrm{x} 40$ ) and (D) (magnification $\mathrm{x} 400$ ), respectively. The expression of claudin-4 in IGC less than in normal tissues is shown in (E) (magnification $\times 200$ ) and (F) (magnification $\times 400$ ), respectively. The expression of claudin-4 in DGC less than in normal tissues is shown in (G) (magnification $\mathrm{x} 200$ ). $\mathrm{T}$, tumor tissues; N, normal tissues; S, strong; E, equal; W, weak. 
Table II. Correlations between claudin-4 expression in cancer and normal tissues and clinicopathological parameters.

\begin{tabular}{|c|c|c|c|c|}
\hline \multirow[t]{2}{*}{ Parameters } & \multicolumn{3}{|c|}{ Claudin-4 (tumor:normal) } & \multirow[t]{2}{*}{ P-value } \\
\hline & Strong N (\%) & Equal N (\%) & Weak N (\%) & \\
\hline \multicolumn{5}{|l|}{ Ages } \\
\hline$\leq 60$ & $24(53 \%)$ & $16(36 \%)$ & $5(11 \%)$ & \multirow[t]{2}{*}{0.6657} \\
\hline$>60$ & $29(61 \%)$ & $16(33 \%)$ & $3(6 \%)$ & \\
\hline \multicolumn{5}{|l|}{ Gender } \\
\hline Male & $33(59 \%)$ & $16(29 \%)$ & $7(12 \%)$ & \multirow[t]{2}{*}{0.1406} \\
\hline Female & $20(54 \%)$ & $16(43 \%)$ & $1(3 \%)$ & \\
\hline \multicolumn{5}{|c|}{ Histological type } \\
\hline Intestinal & $34(76 \%)$ & $10(22 \%)$ & $1(2 \%)$ & \multirow[t]{2}{*}{0.0011} \\
\hline Diffuse & $19(40 \%)$ & $22(46 \%)$ & $7(14 \%)$ & \\
\hline \multicolumn{5}{|c|}{ Depth of wall invasion } \\
\hline $\mathrm{T} 1$ & $12(52 \%)$ & $9(39 \%)$ & $2(9 \%)$ & \multirow{4}{*}{0.6512} \\
\hline $\mathrm{T} 2$ & $4(36 \%)$ & $5(46 \%)$ & $2(18 \%)$ & \\
\hline T3 & $30(64 \%)$ & $14(30 \%)$ & $3(6 \%)$ & \\
\hline $\mathrm{T} 4$ & $7(58 \%)$ & $4(33 \%)$ & $1(9 \%)$ & \\
\hline \multicolumn{5}{|c|}{ Lymph node metastasis } \\
\hline- & $15(46 \%)$ & $15(46 \%)$ & $3(9 \%)$ & \multirow[t]{2}{*}{0.1543} \\
\hline+ & $38(64 \%)$ & $17(28 \%)$ & $5(8 \%)$ & \\
\hline \multicolumn{5}{|c|}{ Differentiation } \\
\hline Well & $8(80 \%)$ & $2(20 \%)$ & $0(0 \%)$ & \multirow{3}{*}{0.6529} \\
\hline Moderate & $16(50 \%)$ & $13(41 \%)$ & $3(9 \%)$ & \\
\hline Poor & $29(57 \%)$ & $17(33 \%)$ & $5(10 \%)$ & \\
\hline
\end{tabular}

The results of immunostaining in tumor and normal tissues were divided into three groups: Strong, rank of tumor tissue >rank of normal tissue; equal, rank of tumor tissue $=$ rank of normal tissue; weak, rank of tumor tissue $<$ rank of normal tissue.

tissue) (Fig. 2C and D), and weak (rank of tumor tissue $<$ rank of normal tissue) (Fig. 2E and F). The overexpression of claudin-4 in IGC and DGC greater than in normal tissues is shown in Fig. 2A and B, respectively. The expression of claudin-4 in IGC and DGC equal to that in normal tissues is shown in Fig. 2C and D, respectively. The expression of claudin-4 in IGC less than in normal tissues is shown in Fig. $2 \mathrm{E}$ and $\mathrm{F}$, respectively. The expression of claudin-4 in DGC less than in normal tissues is shown in Fig. 2G.

Relation between claudin-4 expression and clinicopathological parameters. The relation between claudin-4 expression and the clinicopathological parameters of the patients is shown in Table II. The expression of claudin-4 in IGC is divided into three groups: strong, equal and weak (tumor: normal tissues) and their frequencies were $76 \%$, $22 \%$, and $2 \%$, respectively. In comparison the frequency of claudin-4 expression in DGC was $40 \%, 46 \%$ and $14 \%$, respectively. The overexpression of claudin- 4 was greater in IGC than in DGC ( $p=0.0011)$. Except for histological type, the expression of claudin-4 was not associated with age, gender, depth of wall invasion, lymph node metastasis and differentiation.

\section{Discussion}

In this study, expression of claudin-4 was studied in 93 cases of gastric cancer. The comparison of claudin- 4 in two histologically distinct types of gastric cancer showed that DGC had lower expression of this protein compared with IGC. Thus, the loss of claudin- 4 expression may be the one phenotypic feature distinguishing the two tumors types in analogy with E-cadherin, the expression of which was also lost in DGC $(29,30)$. The loss of claudins and other tight junction proteins in cancer has been interpreted as a mechanism for the loss of cell adhesion, an important step in the progression of cancer to metastasis. A recent study showed that expression of claudin- 4 in pancreatic cancer cells reduces the invasiveness of these cells (31).

The expression of claudins has been found to be altered in several cancer types. Claudin-1 has been found to be reduced in breast cancer as well as in colon cancer (32-34). The loss 
of claudin-7 is associated with a more aggressive behavior of breast carcinoma and head and neck cancer $(35,36)$. These reports of decreased tight junction protein expression in cancer are consistent with the generally accepted idea that tumorigenesis is accompanied by a disruption of tight junctions, a process that may play an important role in the loss of cohesion, invasiveness, and the lack of differentiation observed in cancer cells. In addition to the down-regulation of protein levels, phosphorylation of tight junction proteins, including claudins, may affect tight junction function in cancer. Phosphorylation of claudin- 1 by mitogen activated protein kinases (37) and protein kinase C (38), as well as phosphorylation of claudin-5 by cyclic AMP-dependent protein kinase $(39,40)$ have been reported. Also, WNK4 kinase has been shown to phosphorylate claudin-3 and claudin-4, and decrease tight junction function (41). Phosphorylation of claudin-3 and claudin- 4 in ovarian cancer cells has also been shown to disrupt tight junctions (42).

Paradoxically, other studies have shown that certain claudin proteins are up-regulated in cancer. Overexpression of claudin-3 and 4 has been shown in breast and ovarian carcinoma $(35,43)$. In pancreatic adenocarcinoma and its precursor lesions, claudin-4 overexpression has been found to be present $(31,44)$. Recent work has shown that, at least in the case of ovarian cells, the expression of claudin- 3 and claudin-4 may lead to an increase in invasion, motility, and cell survival (45), all characteristics important for metastasis. Consistent with these in vitro findings is a report that claudin-4 expression in pancreatic intraductal papillary mucinous neoplasms was associated with a more invasive phenotype (46). Claudin-3 and claudin-4 are receptors for the Clostridium perfringens enterotoxin (CPE) (47). CPE is a single polypeptide of $35 \mathrm{kDa}$, which receptor binding causes cytolysis through its effects on membrane permeability. A high expression of claudin-3 and claudin-4 in multiple cancers may thus represent a unique opportunity for innovative therapy using CPE (48). Prostate adenocarcinoma cells expressing claudin- 3 and claudin- 4 have indeed been shown to be sensitive to CPE-mediated cytolysis (49).

The patterns of claudin-4 expression in the various cancers were diverse and controversial. The reasons for the up-regulation or down-regulation in tumorigenesis were unclear. We found the overexpression of claudin-4 in IGC and in general, liver metastasis was frequently seen in IGC (50). The overexpression of claudin-4 may be associated with the distal metastasis of gastric cancer. A recent report showed that claudin-4 expression was associated with increased matrix metalloproteinase- 2 activity in ovarian epithelial cells and enhanced cell invasion (45). Otherwise, the less expression of claudin-4 in DGC correlated well with the general observation that peritoneal metastasis was frequently seen in DGC (50). The decreased claudin-4 expression may be associated with proximal invasion via the loss of the tight junction.

\section{Acknowledgements}

This study was supported by a grant NSC93-2314-B-182-055 from the National Science Council and a grant CMRPG32050 from the Chang Gung Memorial Hospital, Taiwan.

\section{References}

1. Hanahan D and Weinberg RA: The hallmarks of cancer. Cell 100: 57-70, 2000

2. Lauren P: The two histological main types of gastric carcinoma: diffuse and so-called intestinal-type of carcinoma. An attempt at histochemical classification. Acta Pathol Microbiol Scand 64: $31-49,1965$

3. Hughes NR and Bhathal PS: Gastric mucous neck cell and intestinal goblet cell phenotypes in gastric adenocarcinoma. J Clin Pathol 50: 741-748, 1997.

4. Tahara E: Genetic pathways of two types of gastric cancer. IARC Sci Publ 157: 327-349, 2004.

5. Nardone G: Molecular basis of gastric carcinogenesis. Aliment Pharmacol Ther 17 (suppl 2): 75-81, 2003.

6. Fiocca R, Luinetti O, Villani L, et al: Molecular mechanisms involved in the pathogenesis of gastric carcinoma: interactions between genetic alterations, cellular phenotype and cancer histotype. Hepatogastroenterology 48: 1523-1530, 2001.

7. Tahara E, Semba S and Tahara H: Molecular biological observations in gastric cancer. Semin Oncol 23: 307-315, 1996.

8. Tahara E: Molecular mechanism of stomach carcinogenesis. J Cancer Res Clin Oncol 119: 265-272, 1993.

9. Tamura G, Kihana T, Nomura K, et al: Detection of frequent p53 gene mutations in primary gastric cancer by cell sorting and polymerase chain reaction single-strand conformation polymorphism analysis. Cancer Res 51: 3056-3058, 1991

10. Yokozaki H, Kuniyasu H, Kitadai Y, et al: p53 point mutations in primary human gastric carcinomas. J Cancer Res Clin Oncol 119: 67-70, 1992.

11. Yokozaki H, Kuniyasu H, Semba S, et al: Molecular bases of human stomach carcinogesis. In: Molecular Pathology of Gastroenterological Cancer: Application to Clinical Practice. Tahara E (ed). Springer-Verlag, Tokyo, pp55-70, 1997.

12. Uchino S, Tsuda H, Noguchi M, et al: Frequent loss of heterozygosity at the DCC locus in gastric cancer. Cancer Res 52: 3099-3102, 1992

13. Kuniyasu H, Yasui W, Kitadai Y, et al: Frequent amplification of the c-met gene in scirrhous type stomach cancer. Biochem Biophys Res Commun 189: 227-232, 1992.

14. Tahara E, Yokozaki H and Yasui W: Stomach genetic and epigenetic alterations of preneoplastic and neoplastic lesions. In: Molecular Pathology of Early Cancer. Srivastava S, Henson DE and Gazdar A (eds). IOS Press, Amsterdam, pp341-361, 1998.

15. Caca K, Kolligs FT, Ji X, et al: Beta- and gamma-catenin mutations, but not E-cadherin inactivation, underlie T-cell factor/lymphoid enhancer factor transcriptional deregulation in gastric and pancreatic cancer. Cell Growth Differ 10: 369-376, 1999.

16. Becker KF, Atkinson MJ, Reich U, et al: E-cadherin gene mutations provide clues to diffuse type gastric carcinomas. Cancer Res 54: 3845-3852, 1994.

17. Gonzalez-Mariscal L, Betanzos A, Nava P, et al: Tight junction proteins. Prog Biophys Mol Biol 81: 1-44, 2003.

18. Mitic LL, Van Itallie CM and Anderson JM: Molecular physiology and pathophysiology of tight junctions I. Tight junction structure and function: lessons from mutant animals and proteins. Am J Physiol Gastrointest Liver Physiol 279: G250-254, 2000.

19. Tsukita S, Furuse M and Itoh M: Multifunctional strands in tight junctions. Nat Rev Mol Cell Biol 2: 285-293, 2001.

20. Matter K and Balda MS: Signalling to and from tight junctions. Nat Rev Mol Cell Biol 4: 225-236, 2003.

21. Langbein L, Pape UF, Grund C, et al: Tight junction-related structures in the absence of a lumen: occludin, claudins and tight junction plaque proteins in densely packed cell formations of stratified epithelia and squamous cell carcinomas. Eur J Cell Biol 82: 385-400, 2003

22. Martin TA and Jiang WG: Tight junctions and their role in cancer metastasis. Histol Histopathol 16: 1183-1195, 2001.

23. Itoh M and Bissell MJ: The organization of tight junctions in epithelia: implications for mammary gland biology and breast tumorigenesis. J Mammary Gland Biol Neoplasia 8: 449-462, 2003

24. Mullin JM: Epithelial barriers, compartmentation, and cancer. Sci STKE 216: pe2, 2004.

25. Wu CM, Lee YS, Wang TH, et al: Identification of differential gene expression between intestinal and diffuse gastric cancer using cDNA microarray. Oncol Rep 15: 57-64, 2006. 
26. Schraml P, Bucher C, Bissig H, et al: Cyclin E overexpression and amplification in human tumours. J Pathol 200: 375-382, 2003.

27. Ravn V, Havsteen $\mathrm{H}$ and Thorpe SM: Immunohistochemical evaluation of estrogen and progesterone receptors in paraffinembedded, formalin-fixed endometrial tissues: comparison with enzyme immunoassay and immunohistochemical analysis of frozen tissue. Mod Pathol 11: 709-715, 1998.

28. Ravn V, Rasmussen BB, Hojholt L, et al: Reproducibility of subjective immunohistochemical estrogen- and progesteronereceptor determination in human endometrium. Pathol Res Pract 189: 1015-1022, 1993.

29. Machado JC, Carneiro F, Beck S, et al: E-cadherin expression is correlated with the isolated cell/diffuse histotype and with the features of biological aggressiveness of gastric carcinoma. Int J Surg Pathol 6: 135-144, 1998.

30. Mayer B, Johnson JP, Leitl F, et al: E-cadherin expression in primary and metastatic gastric cancer: down-regulation correlates with cellular dedifferentiation and glandular disintegration. Cancer Res 53: 1690-1695, 1993.

31. Michl P, Barth C, Buchholz M, et al: Claudin-4 expression decreases invasiveness and metastatic potential of pancreatic cancer. Cancer Res 63: 6265-6271, 2003.

32. Kramer F, White K, Kubbies M, et al: Genomic organization of claudin-1 and its assessment in hereditary and sporadic breast cancer. Hum Genet 107: 249-256, 2000.

33. Tokes AM, Kulka J, Paku S, et al: Claudin-1, -3 and -4 proteins and mRNA expression in benign and malignant breast lesions: a research study. Breast Cancer Res 7: R296-305, 2005.

34. Resnick MB, Konkin T, Rou J, et al: Claudin-1 is a strong prognostic indicator in stage II colonic cancer: a tissue microarray study. Mod Pathol 18: 511-518, 2005.

35. Kominsky SL, Argani P, Korz D, et al: Loss of the tight junction protein claudin-7 correlates with histological grade in both ductal carcinoma in situ and invasive ductal carcinoma of the breast. Oncogene 22: 2021-2033, 2003.

36. Al Moustafa AE, Alaoui-Jamali MA, Batist G, et al: Identification of genes associated with head and neck carcinogenesis by cDNA microarray comparison between matched primary normal epithelial and squamous carcinoma cells. Oncogene 21: 2634-2640, 2002.

37. Fujibe M, Chiba H, Kojima T, et al: Thr $^{203}$ of claudin-1, a putative phosphorylation site for MAP kinase, is required to promote the barrier function of tight junctions. Exp Cell Res 295: 36-47, 2004

38. Nunbhakdi-Craig V, Machleidt T, Ogris E, et al: Protein phosphatase $2 \mathrm{~A}$ associates with and regulates atypical $\mathrm{PKC}$ and the epithelial tight junction complex. J Cell Biol 158: 967-978, 2002.
39. Ishizaki T, Chiba H, Kojima T, et al: Cyclic AMP induces phosphorylation of claudin-5 immunoprecipitates and expression of claudin-5 gene in blood-brain-barrier endothelial cells via protein kinase A-dependent and -independent pathways. Exp Cell Res 290: 275-288, 2003.

40. Soma T, Chiba H, Kato-Mori Y, et al: $\operatorname{Thr}(207)$ of claudin-5 is involved in size-selective loosening of the endothelial barrier by cyclic AMP. Exp Cell Res 300: 202-212, 2004.

41. Yamauchi K, Rai T, Kobayashi K, et al: Disease-causing mutant WNK4 increases paracellular chloride permeability and phosphorylates claudins. Proc Natl Acad Sci USA 101: 4690-4694, 2004.

42. D'Souza T, Agarwal R and Morin PJ: Phosphorylation of claudin-3 at threonine 192 by cAMP-dependent protein kinase regulates tight junction barrier function in ovarian cancer cells. J Biol Chem 280: 26233-26240, 2005.

43. Kominsky SL, Vali M, Korz D, et al: Clostridium perfringens enterotoxin elicits rapid and specific cytolysis of breast carcinoma cells mediated through tight junction proteins claudin 3 and 4. Am J Pathol 164: 1627-1633, 2004.

44. Terris B, Blaveri E, Crnogorac-Jurcevic T, et al: Characterization of gene expression profiles in intraductal papillary mucinous tumors of the pancrease. Am J Pathol 160: 1745-1754, 2002.

45. Agarwal R, D'Souza T, and Morin PJ: Claudin-3 and claudin-4 expression in ovarian epithelial cells enhances invasion and is associated with increased matrix metalloproteinase-2 activity. Cancer Res 65: 7378-7385, 2005.

46. Sato N, Fukushima N, Maitra A, et al: Gene expression profiling identifies genes associated with invasive intraductal papillary mucinous neoplasms of the pancreas. Am J Pathol 164: 903-914, 2004

47. Katahira J, Sugiyama H, Inoue N, et al: Clostridium perfringens enterotoxin utilizes two structurally related membrane proteins as functional receptors in vivo. J Biol Chem 272: 26652-26658, 1997.

48. Michl P and Gress TM: Bacterial and bacterial toxins as therapeutic agents for solid tumors. Curr Cancer Drug Targets 4: 689-702, 2004.

49. Long H, Crean CD, Lee WH, et al: Expression of Clostridium perfringens enterotoxin receptors claudin-3 and claudin-4 in prostate cancer epithelium. Cancer Res 61: 7878-7881, 2001.

50. Umehara Y, Kimura T, Yoshida M, et al: Metastatic mode of gastric carcinoma by flow cytometric and clinicopathologic parameters. Clin Exp Metastasis 10: 19-24, 1992. 